\title{
Adiponectin ameliorates angiotensin II-induced vascular endothelial damage
}

\author{
Zuo Zhi • Zuo Pengfei • Tian Xiaoyi • Ma Genshan
}

Received: 21 October 2013 / Revised: 11 January 2014 / Accepted: 22 January 2014 / Published online: 13 February 2014

(C) Cell Stress Society International 2014

\begin{abstract}
Adiponectin is an adipocyte-specific adipocytokine that possesses anti-atherogenic and anti-diabetic properties. It has been shown to have a beneficial effect on the cardiovascular system, but it remains to be elucidated whether adiponectin has a therapeutic effect on vascular damage induced by the potential vasoactive substance angiotensin II (Ang II). In this study, the effects of adiponectin on Ang IIinduced vascular endothelial damage were investigated. In cultured human umbilical vein endothelium cells, Ang II stimulation increased generation of ROS and 4-hydroxy-2nonenal, both of which were clearly restored by administration of adiponectin. In addition, administration of adiponectin was found to increase cell viability and prevent apoptosis. Our results also demonstrate that the protective effects of adiponectin against Ang II-induced vascular endothelial damage are dependent on the binding of adiponectin to its cell surface receptor 1. Importantly, we found that adiponectin treatment modulates the apoptotic pathway by reducing the expression of LOX-1, up-regulating both cIAP-1 and the ratio of Bcl-2/Bax. Finally, our data displayed that the protective effects of adiponectin against Ang II cytotoxicity depend on AMPK activation mediated by the endosomal adaptor protein, adaptor protein with phosphotyrosine binding, pleckstrin homology domains, and leucine zipper motif.
\end{abstract}

Z. Zhi $\cdot$ Z. Pengfei $\cdot$ M. Genshan $(\bowtie)$

Department of Cardiology, Zhongda Hospital, Medical School of

Southeast University, Dingjiaqiao Road No. 87,

Nanjing 210009, Jiangsu Province, China

e-mail: gsma666@hotmail.com

Z. Zhi

e-mail: zuozhi1988@gmail.com

T. Xiaoyi

Department of Cardiology, Huai' an Municipal First People's

Hospital, Huai'an 223400, Jiangsu Province, China
Keywords Adiponectin · Angiotensin II · Apoptosis · Oxidative stress $\cdot$ LOX-1

\section{Introduction}

Endothelial dysfunction plays an initial role in the development of cardiovascular diseases and their pathogenesis (Davignon and Ganz 2004). Angiotensin II (Ang II), the main effector peptide of the renin-angiotensin system (RAS), has been implicated in endothelial dysfunction via the activation of specific Ang II receptors (Xue et al. 2012). The process of endothelial dysfunction induced by Ang II has been implicated in a variety of cardiovascular diseases including atherosclerosis, hypertension, left ventricular hypertrophy, myocardial infarction, and heart failure (Dimmeler et al. 1997). Both academic scientists and clinical physicians have put increasingly more effort into understanding the mechanisms responsible for Ang II's ability to act as a mediator of endothelial apoptosis. It has been reported that activation of Ang II signaling triggers the production of reactive oxygen species (ROS) by inducing multiple downstream pathways in endothelial cells (Sata and Fukuda 2010). In addition, activation of proapoptotic protein has been suggested to be involved in Ang II-induced apoptosis related to endothelial dysfunction (Kim and Day 2012). Blockage of Ang II signaling by specific antagonists of the Ang II type 1 receptor has been reported to protect the retinal vasculature against hypertensive vascular injury by inhibiting vascular cell apoptosis (Yang et al. 2010).

Adipose tissue is now known to secrete several proteins and bioactive peptides. Among them, adiponectin is probably the most abundantly secreted adipokine in serum. Adiponectin has been reported to possess insulin-sensitizing, antiinflammatory, and antioxidant properties (Kadowaki et al. 2006). Serum adiponectin levels are decreased in a variety of chronic diseases including obesity, obesity-related insulin 
resistance, T2DM, and coronary artery disease (Lam and $\mathrm{Xu}$ 2005). Importantly, adiponectin is reported to protect against major obesity-related pathologies including hypertension, atherosclerosis, non-alcoholic fatty liver, non-alcoholic steatohepatitis, heart failure, airway inflammation, and some cancers (Wang et al. 2008). There is now a general agreement that adiponectin exerts many of its cellular effects primarily by binding to two receptor isoforms (adiponectin receptors type 1 (AdipoR1) and adiponectin receptors type 2 (AdipoR2)). Adaptor protein with phosphotyrosine binding, pleckstrin homology domains and a leucine zipper motif (APPL1) binds to adiponectin receptors and mediates both adiponectin signaling and function (Mao et al. 2006). Most physiological actions of adiponectin are mediated by phosphorylation of adenosine monophosphateactivated protein kinase (AMPK) at threonine 172.

Endothelial cells are central to the homeostatic processes of the vascular wall, including effective immune response and the repair of injured vessels. Drugs interfering with the RAS system including Ang II have been considered as an important pharmacological target in various cardiovascular diseases. Although the effects of adiponectin on glucose metabolism have been well documented (Akingbemi et al. 2013), the exact role of adiponectin in endothelial cells against Ang II-induced cell apoptosis and the underlying molecular mechanisms have not yet been fully clarified. In addition, it is unknown whether human umbilical vein endothelial cell (HUVEC) apoptosis is associated with adipoRs and AMPK (Zhao et al. 2011). The aim of the present study is to understand the mechanisms underlying suppression of apoptosis by adiponectin in Ang II-treated HUVECs. We hypothesize that adiponectin is protective against Ang II toxicity in endothelial cells. In this communication, we report that adiponectin has the ability to protect against vascular endothelial damage in HUVEC under Ang II toxicity as well as its underlying mechanisms of protection.

\section{Materials and methods}

Cell culture, treatment, and transfection

HUVECs from Lonza (Walkersville, USA) were cultured in EBM-2 media with supplemental growth factors according to manufacturer's instructions (Zhou et al. 2012). Cells were pretreated with adiponectin (Sigma-Aldrich, USA) at 1, 5, or $10 \mu \mathrm{g} / \mathrm{ml}$ for $4 \mathrm{~h}$ before adding $1-\mu \mathrm{M}$ Ang II. Small interfering RNA (siRNA) for AdipoR1 and AdipoR2, APPL1, AMPK, and scrambled RNA (negative control) were from Invitrogen, USA. Cells were transfected with target siRNAs using Lipofectamine 2000 (Invitrogen, USA) according to the manufacturer's instructions (Shi H et al. 2013).
Intracellular ROS determination

Levels of intracellular ROS production were determined by fluorescence dye $2^{\prime}, 7^{\prime}$-dichlorofluorescein-diacetate (DCFHDA) (Sigma-Aldrich, USA). Briefly, after the indicated treatment, cells were loaded with $15-\mu \mathrm{M}$ DCFH-DA and incubated in a $\mathrm{CO}_{2}$ incubator at $37^{\circ} \mathrm{C}$ for $1 \mathrm{~h}$. Green fluorescence signals were observed using a fluorescence microscope. Average fluorescence intensity, as analyzed by Image-Pro Plus software, was used to represent levels of intracellular ROS.

4-hydroxy-2-nonenal immunofluorescence

Levels of 4-hydroxy-2-nonenal (4-HNE) found in HUVECs were used to index lipid peroxidation. Briefly, after being fixed in $4 \%$ paraformaldehyde for $10 \mathrm{~min}$ at room temperature (RT), HUVECs were permeabilized with $0.4 \%$ Triton X-100 and blocked with both $5 \%$ BSA and $2.5 \%$ FBS in PBST. After that, cells were incubated with anti-4-HNE antibodies (Cell Signaling, USA) for $2 \mathrm{~h}$ at RT, followed by incubation with Alexa-594 conjugated secondary antibodies (Invitrogen, USA) for $1 \mathrm{~h}$ at RT.

\section{Cell viability}

Cell viability of HUVECs was determined by MTT reduction assay (He et al. 2006). Upon completion of the indicated treatment, MTT was added to each well, with a final concentration of $1.0 \mathrm{mg} / \mathrm{ml}$, and incubated for $4 \mathrm{~h}$ at $37^{\circ} \mathrm{C}$. DMSO was used to dissolve the formed formazan crystals. Cell viability was indexed by OD values measured at $570 \mathrm{~nm}$ using a microplate reader.

\section{Determination of lactate dehydrogenase}

Cell membrane integrity was indexed by the release of lactate dehydrogenase (LDH) into the medium. Briefly, when the indicated treatment had been completed, measurements of LDH activity in the medium were taken using a commercially available assay kit (Roche Applied Science) according to the manufacturer's instructions. Absorbance was read at $490 \mathrm{~nm}$ and calculated as the percentage of cell death (Sheng et al. 2009).

Western blot analysis

Following the indicated treatment and transfection, HUVECs were lysed in lysis buffer $(25 \mathrm{mmol} / \mathrm{L}$ Tris- $\mathrm{HCl}, \mathrm{pH} 8.0,1 \%$ Nonidet-P40, $0.5 \%$ sodium deoxycholate, $0.1 \%$ sodium dodecyl sulfate (SDS), and $125 \mathrm{mmol} / \mathrm{L} \mathrm{NaCl}$ ) with complete protease inhibitor cocktail (Roche, Mannheim, Germany). Protein concentration was determined by a bicinchoninic acid 

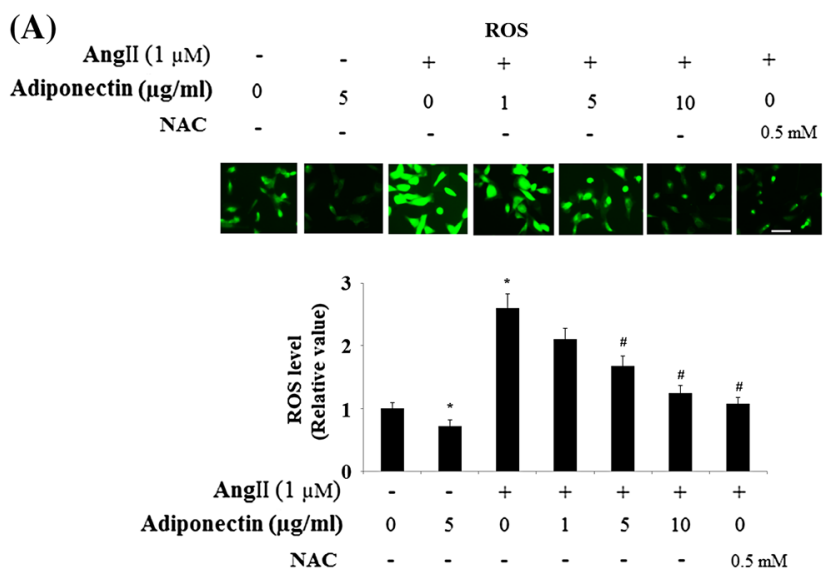

Fig. 1 Effects of adiponectin on Angiotensin II (Ang II)-induced oxidative stress. Antioxidant N-acetyl cysteine (NAC) was used as a positive control. Scale bar $100 \mu \mathrm{m}$. a ROS was measured by DCFH-DA. Representative fluorescence photos of intracellular ROS show increased intracellular ROS accumulation in Ang II-treated cells, which was inhibited by adiponectin in a dose-dependent manner (ANOVA $* p<0.001$ vs. non-

kit (Sigma-Aldrich, USA). Samples of protein extracts were resolved by SDS-polyacrylamide gel electrophoresis and transferred to polyvinylidene fluoride membranes. After blocking with $5 \%$ non-fat milk for $2 \mathrm{~h}$ at RT, membranes were incubated for $3 \mathrm{~h}$ at RT with primary antibodies followed by incubation with the appropriate secondary antibodies (Santa Cruz Biotechnology). Signals were determined using an enhanced chemiluminescence detection kit (Amersham Biosciences, USA) (Sheng et al. 2012).

\section{TUNEL assay and DAPI staining}

HUVECs were plated on Lab-Tek ${ }^{\circledR}$ Chamber Slides (Nalge Nunc, Naperville, IL, USA) and incubated in the indicated medium. Upon completion of the indicated treatment, apoptosis was determined by terminal deoxynucleotidyl transferase dUTP nick end labeling (TUNEL) assay using a commercially available kit (Promega, Madison, WI, USA). Manufacturer's protocols were followed. Nuclei were counterstained with 4,6-

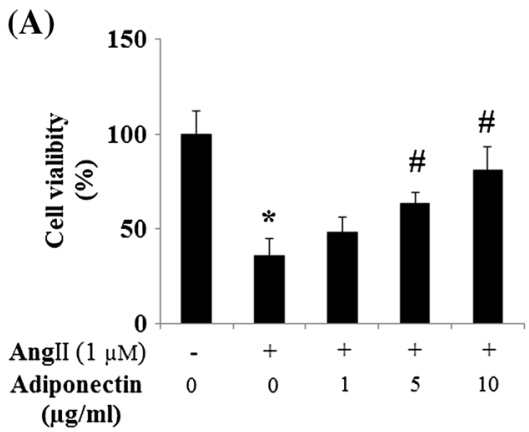

Fig. 2 Adiponectin protects HUVECs against Ang II-induced cell death. a Adiponectin prevents HUVEC cell death induced by Ang II. Cell viability was measured by MTT (ANOVA was performed: ANOVA ${ }^{*} p<0.001$ vs. non-treated control; $\# p<0.001$ vs. Ang II-treated group,
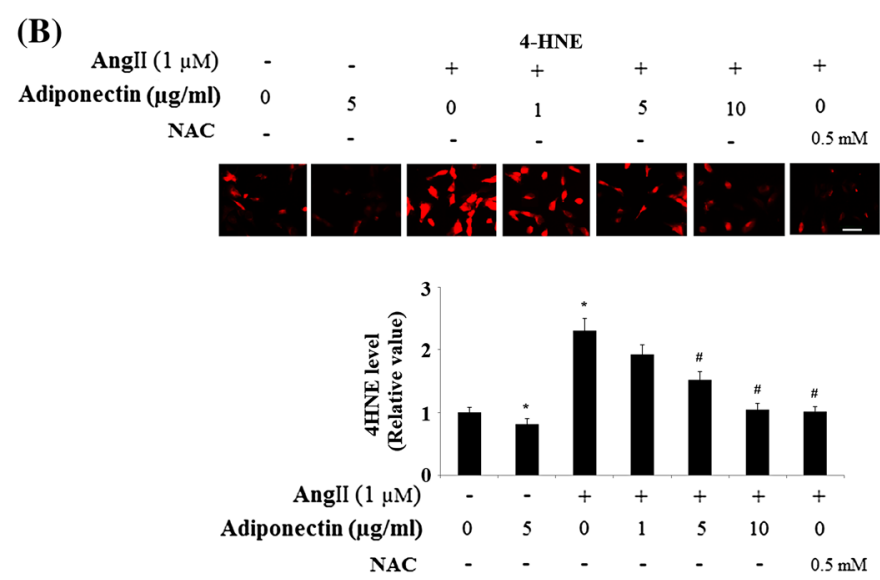

treated control; $\# p<0.001$ vs. Ang II-treated group, $n=3-5$ ); $\mathbf{b}$, Representative fluorescence photos of intracellular 4-HNE show increased intracellular 4-HNE accumulation in Ang II-treated cells, which was inhibited by adiponectin in a dose-dependent manner (ANOVA $* p<0.001$ vs. nontreated control; $\# p<0.001$ vs. Ang II-treated group, $n=3-5$ )

diamidino-2-phenylindole (DAPI). Fluorescence signals were taken using a fluorescence microscope and a digital camera.

Statistical analysis

All statistical analyses were carried out using the statistical analysis software package SPSS 19 (SPSS, Munich, Germany). Data are presented as mean \pm standard deviation. A univariate analysis of variance (ANOVA) was calculated to compare the data in different groups. $P<0.05$ was considered as a statistical significance.

\section{Results}

Oxidative stress is one of the main causes of Ang II toxicity. Thus, we investigated oxidative stress patterns in our experiments. Altered levels of ROS and 4-HNE are the two essential parameters of oxidative stress. To study the possible roles of

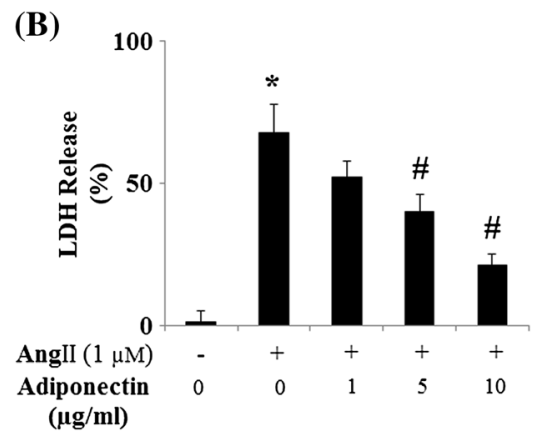

$n=4)$; b Adiponectin prevents LDH release in HUVECs induced by Ang II. LDH release was measured by a commercial kit (ANOVA * $p<0.001$ vs. non-treated control; $\# p<0.001$ vs. Ang II-treated group, $n=3-5$ ) 
Fig. 3 Adiponectin prevents Ang II-induced apoptosis in HUVECs. Cells were stained using a TUNEL assay kit. Nuclear DNA was stained with DAPI. Scale bar $50 \mu \mathrm{m}$. a Representative stainings; b. quantitative analysis of the apoptotic rate (ANOVA ${ }^{*} p<0.001$ vs. non-treated control; $\# p<0.001$ vs. Ang II-treated group, $n=6$ )
(A)

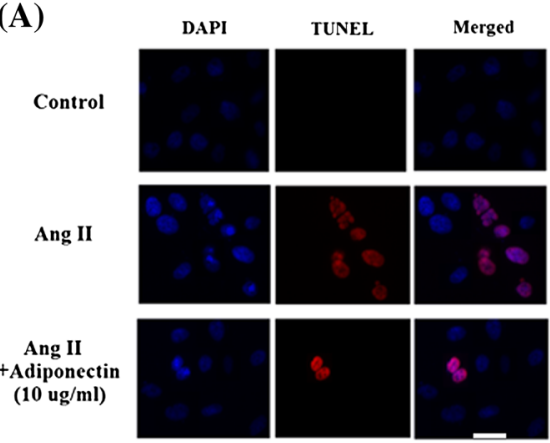

(B)

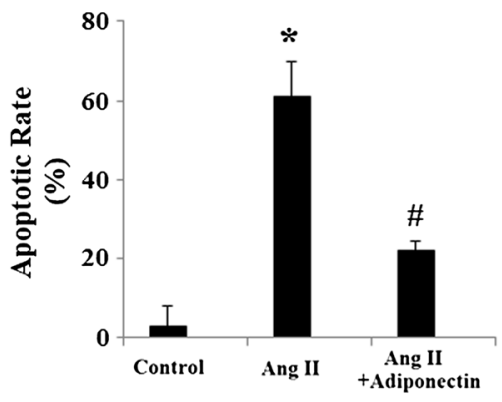

adiponectin in regulating oxidative stress induced by Ang II, we measured intracellular ROS and 4-HNE levels in HUVECs after incubation with Ang II and adiponectin. DCFH-DA fluorescence staining experiments indicated that intracellular ROS levels in Ang II-treated cells were significantly higher than in controls and that this could be attenuated by adiponectin in a dose-dependent manner from 1 to $10 \mu \mathrm{g} / \mathrm{ml}$ (Fig. 1a). Consistently, Ang II treatment led to increased levels of 4-HNE, but was prevented by adiponectin (Fig. 1b). In addition, treatment with adiponectin reduced the basal levels of ROS (Fig. 1a) and 4-HNE (Fig. 1b). Antioxidant N-acetyl cysteine (NAC) was used as a positive control.

We also measured endothelial cell viability. Both MTT (Fig. 2a) and LDH (Fig. 2b) assays displayed that 24-h treatment with Ang II led to impaired cell viability. Notably, Ang II-induced cell death was effectively prevented by adiponectin treatment in a dose-dependent manner.

Ang II has been shown to regulate apoptosis in multiple cell lines. In order to determine whether adiponectin can have a direct effect upon Ang II-induced apoptosis, TUNEL staining was used to detect apoptosis following Ang II and adiponectin treatment. As shown in Fig. 3a, Ang II significantly promoted apoptosis in cells. However, adiponectin treatment ameliorated these patterns of apoptosis. The apoptotic rate of HUVECs was shown in Fig. 3b. Mechanistically, our results demonstrate that caspase- 3 is activated in HUVECs after AngII treatment and that the activation of caspase- 3 is significantly inhibited by adiponectin treatment (Fig. 4a, b).
Cleavage of poly (ADP-ribose) polymerase (PARP) is catalyzed by caspase-3. Our data also indicated that AngII treatment increased the levels of cleaved PARP, which was prevented by adiponectin (Fig. 4a, b).

Previous studies demonstrate that adiponectin acts by binding to its associated receptors, AdipoR1 and AdipoR2 (Yamauchi and Kadowaki 2008). In order to confirm that the observed protective effects of adiponectin against Ang II toxicity in HUVECs were a result of adiponectin binding to its cell surface receptors, we knocked down the expression of AdipoR1 and AdipoR2 by RNA interference in HUVECs. Successful knockdown was confirmed by Western blot analysis (Fig. 5a, c). Results show that the protective effects of adiponectin against Ang II-induced cytotoxicity were lost in HUVECs with knockdown of AdipoR1 (Fig. 5b) but not AdipoR2 (Fig. 5d). These data suggest that the protective effects of adiponectin against Ang II-induced cytotoxicity require binding of adiponectin to its cell surface receptor AdipoR1.

Lectin-like, oxidized low-density lipoprotein receptor-1 (LOX-1) has recently been documented as playing a critical role in endothelial apoptosis. It mediates endothelial apoptosis via the down-regulation of multiple anti-apoptotic proteins including Bcl-2 and inhibitory apoptotic protein-1 (cIAP-1) (Li and Mehta 2009). Our results indicate that Ang II treatment led to the up-regulation of LOX-1 expression. However, up-regulation of LOX-1 was significantly inhibited by adiponectin treatment (Fig. 6).
Fig. 4 Effects of adiponectin on the levels of cleaved caspase-3 and PARP. a Representative images of immunoblots for cleaved caspase-3 and PARP; $\mathbf{b}$ Quantitative analysis of cleaved caspase-3 and PARP. (ANOVA $*_{p}<0.01$ vs. non-treatment group; $\# p<0.01$ vs. Ang II-treated group, $n=4$ )
(A)

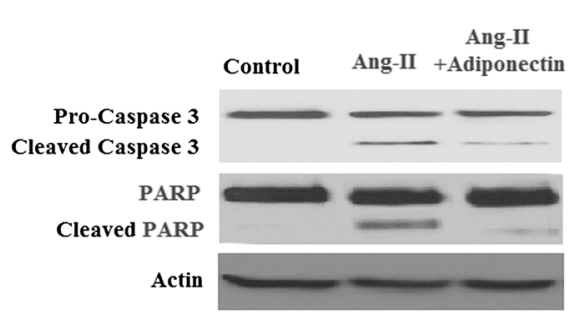

(B)

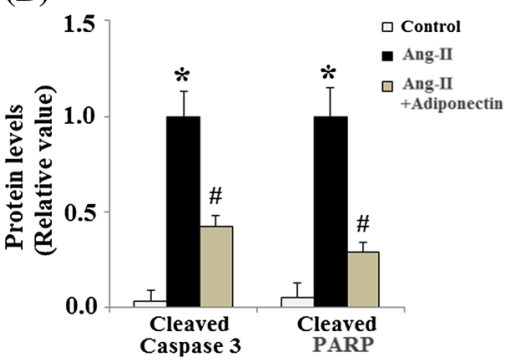


(A)

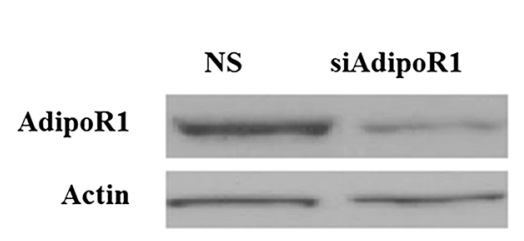

(C)

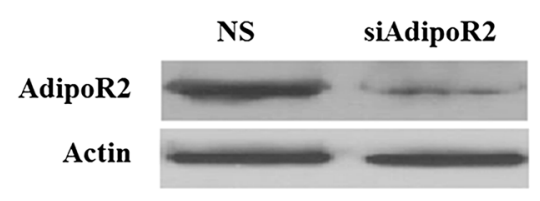

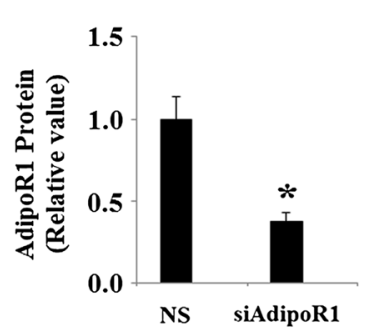

(B)

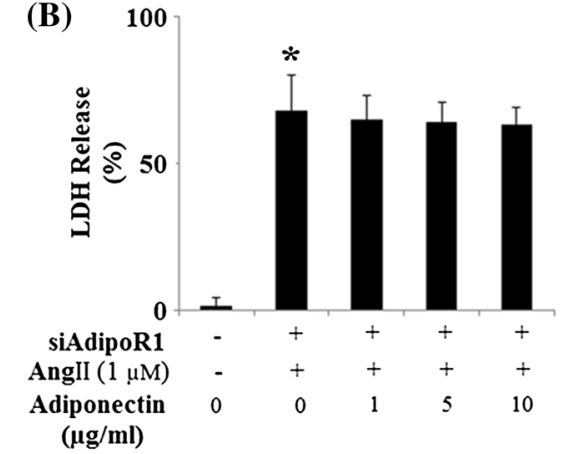

(D)

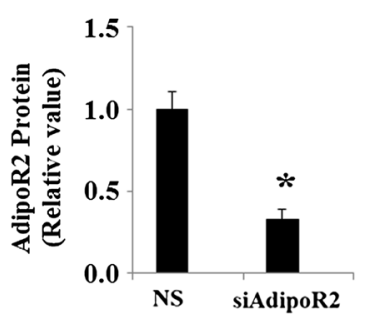

Fig. 5 The protective effects of adiponectin against cytotoxicity of HUVECs under Ang II treatment require binding of adiponectin to its cell surface receptors, AdipoR1 or AdipoR2. NS non-specific RNA; siAdipoR1 small RNA interference of AdipoR1; siAdipoR2 small RNA interference of AdipoR2. a Expression of adiponectin receptor 1 (AdipoR1) was knocked down using small RNA interferences, and western blot results confirmed successful knockdown $\left({ }^{*} p<0.01\right.$ vs. NS group, $n=4)$; $\mathbf{b} \mathrm{LDH}$ release experiment revealed that the protective effects of adiponectin against Ang II cytotoxicity were lost in cells with

Importantly, our results also demonstrated that the protective effects of adiponectin against Ang II-induced upregulation of LOX-1 were lost in HUVECs with knockdown of AdipoR1 (Fig. 7).

Since many of the important cellular functions of adiponectin are regulated by APPL1-mediated AMPK activation, we proceeded to study whether APPL1-dependent AMPK activation is involved in the protective effects of adiponectin against Ang II cytotoxicity. Using HUVECs with knockdown of APPL1 expression by RNA interference (Fig. 8a), we found that the protective effects of adiponectin against cytotoxicity under Ang II treatment were lost in cells with knockdown of APPL1 expression (Fig. 8b). Notably, it was found that the protective effects of adiponectin against Ang II-induced up-regulation of LOX-1 were lost in HUVECs with knockdown of APPL1 (Fig. 8c, d). These results suggest
Fig. 6 Effects of adiponectin on the expression of LOX-1 in HUVECs. a Representative images of immunoblots for LOX1. Expression of $\beta$-actin was used as a loading control; $\mathbf{b}$ Quantitative analysis of LOX-1 (ANOVA $* p<0.01$ vs. nontreatment group; $\# p<0.01$ vs. Ang II-treated, but not adiponectin-treated group, $n=3$ )
(A)

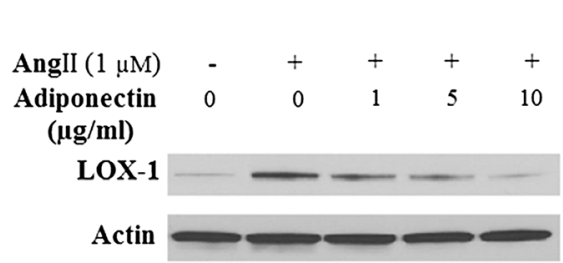

(B)

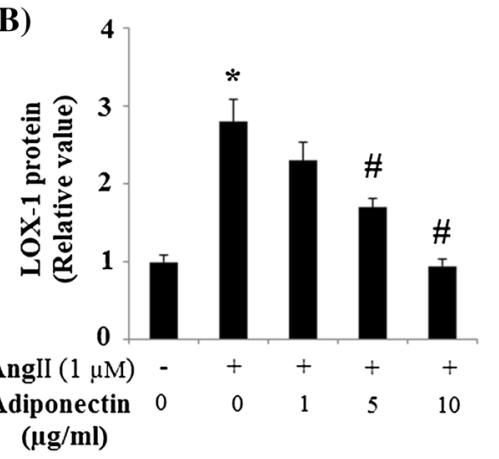


(A)

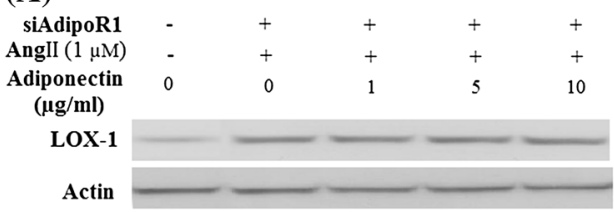

(B)

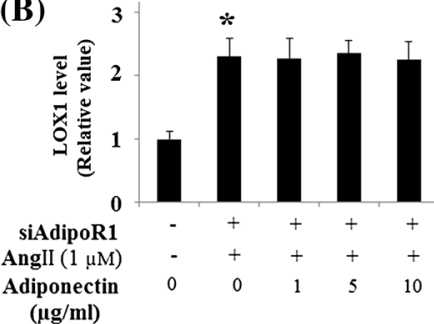

Fig. 7 Effects of adiponectin on the expression of LOX-1 requires AdipoR1. Expression of adiponectin receptor 1 (AdipoR1) was knocked down using small RNA interferences. a Representative images of

that the protective effects of adiponectin against Ang IImediated cytotoxicity under Ang II insult are dependent on APPL1. To further verify that the protective effects of adiponectin against Ang II cytotoxicity are mediated through activation of AMPK, HUVECs were transfected with AMPK siRNA (Fig. 9a). The results show that the protective effects of adiponectin against Ang II cytotoxicity were lost in cells with knockdown of AMPK expression (Fig. 9b). Importantly, our results also demonstrated that the protective effects of adiponectin against Ang II-induced up-regulation of LOX-1 were lost in HUVECs with knockdown of AMPK (Fig. 9c, d). immunoblots for LOX-1. Expression of $\beta$-actin was used as a loading control; b Quantitative analysis of LOX-1 (ANOVA $* p<0.01$ vs. nontreatment group, $n=3$ )

Consistently, we examined the effects of adiponectin on the expression of cIAP-1. Findings show that cIAP-1 was significantly decreased in HUVECs after Ang II treatment, an effect that was markedly prevented by adiponectin treatment (Fig. 10). Since Bax and Bcl-2 have been known to be involved in Ang II-induced apoptosis (Tan et al. 2009), we investigated next the influence of adiponectin on the expression of these two apoptosis regulators. The addition of $1-\mu \mathrm{M}$ Ang II to the cell cultures decreased Bcl-2 but increased Bax (Fig. 11). Accordingly, the Bcl-2/Bax ratio, a survival indicator, was significantly reduced in cells treated with Ang II
(A)

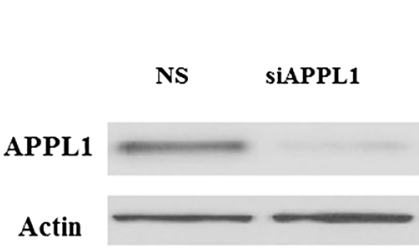

(C)

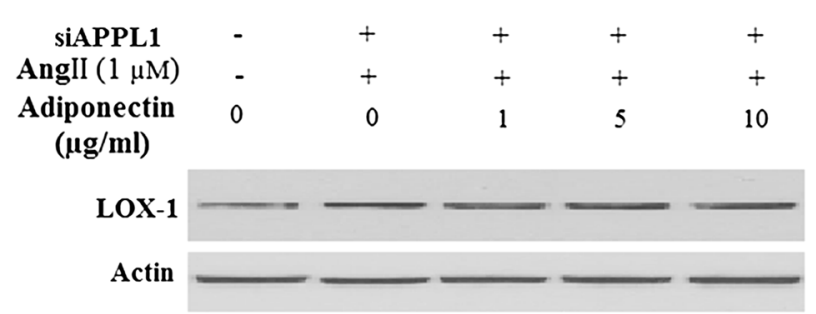

(B)
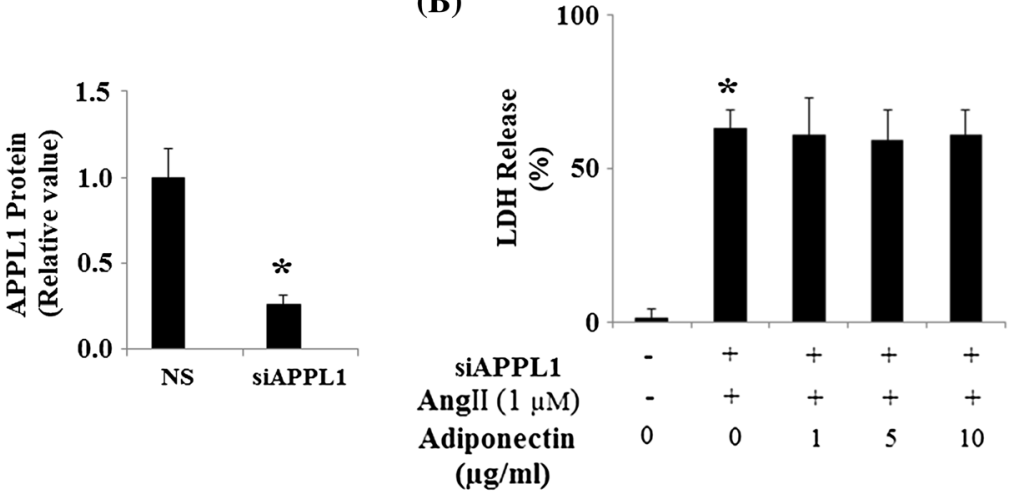

(D)

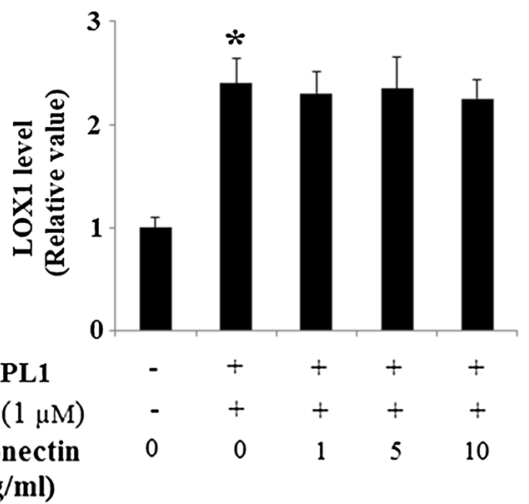

effects of adiponectin against the cytotoxicity of Ang II treatment was lost in cells with knockdown of APPL1 $\left(^{*} p<0.01\right.$ vs. non-treatment group, $n=4)$; c Representative images of immunoblots for LOX-1. Expression of $\beta$-actin was used as a loading control; $\mathbf{d}$ Quantitative analysis of LOX-1 (ANOVA $* p<0.01$ vs. non-treatment group, $n=3$ ) 
(A)
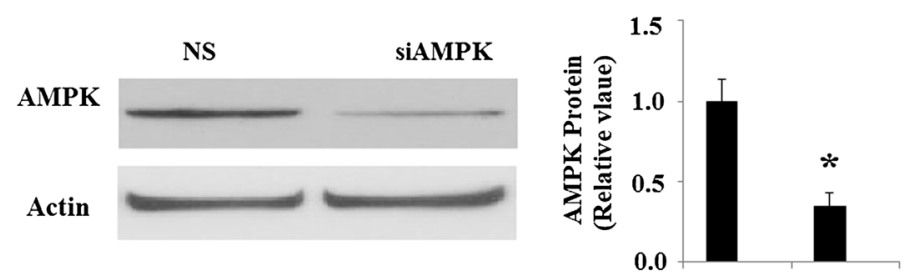

(B)

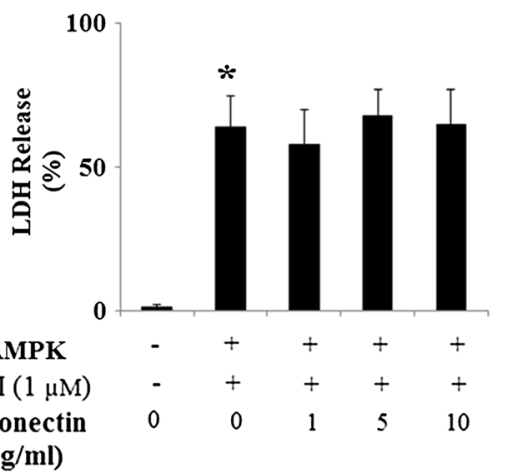

(C)

\begin{tabular}{|c|c|c|c|c|c|}
\hline siAMPK & - & + & + & + & + \\
\hline AngII $(1 \mu \mathrm{M})$ & - & + & + & + & + \\
\hline $\begin{array}{c}\text { Adiponectin } \\
(\mu \mathrm{g} / \mathrm{ml})\end{array}$ & 0 & 0 & 1 & 5 & 10 \\
\hline \multicolumn{6}{|l|}{ LOX1 } \\
\hline Actin & & & & & \\
\hline
\end{tabular}

(D)

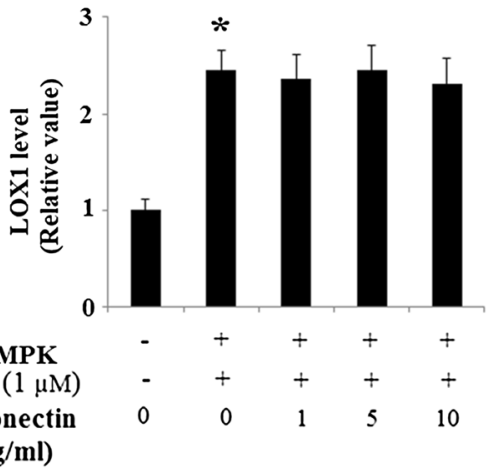

Fig. 9 Protection effects of adiponectin on Ang II-induced cytotoxicity and the expression of LOX-1 requires AMPK. a The expressions of AMPK were knocked down using small RNA interferences, and the western blot result confirmed the successful knockdown $\left({ }^{*} p<0.01\right.$ vs. NS group, $n=4)$; $\mathbf{b}$ LDH assay revealed that the protective effects of

( $p<0.01$ relative to the control cells). In contrast, adiponectin treatment prevented $\mathrm{Bcl}-2 / \mathrm{Bax}$ reduction $(p<0.01$ relative to cells treated with Ang II alone), indicating potential for enhanced anti-apoptotic activity.

\section{Discussion}

The present study demonstrates that adiponectin works to protect HUVECs against Ang II-induced cytotoxicity by

(A)

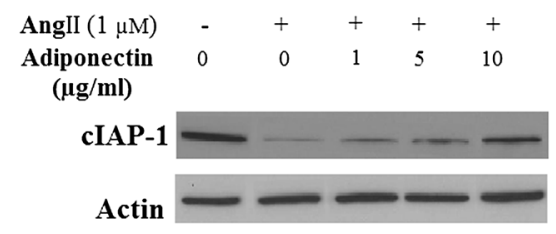

Fig. 10 Effects of adiponectin on the expression of cIAP-1 in HUVECs. a Representative images of immunoblots for cIAP-1. The expression of $\beta$-actin was used as a loading control; b Quantitative analysis of cIAP-1 adiponectin against the cytotoxicity of Ang II treatment was lost in cells with knockdown of AMPK $\left({ }^{*} p<0.01, n=4\right)$; $\mathbf{c}$ Representative images of immunoblots for LOX-1. Expression of $\beta$-actin was used as a loading control; d Quantitative analysis of LOX-1 (ANOVA * $p<0.01$ vs. nontreatment group, $n=3$ )

attenuating oxidative stress, increasing cell viability, and preventing apoptosis. In addition, the protective effects of adiponectin depend on its ability to bind to its associated receptors, AdipoR1 and AdipoR2. Importantly, our results demonstrate that adiponectin treatment modulates the apoptotic pathway by reducing the expression of LOX-1 and upregulating both cIAP-1 expression and the Bcl-2/Bax ratio.

Vascular endothelial cells play a critical role in the regulation of many physiological processes such as coagulation, inflammation, and arterial tone (Lee et al. 2012). Endothelial

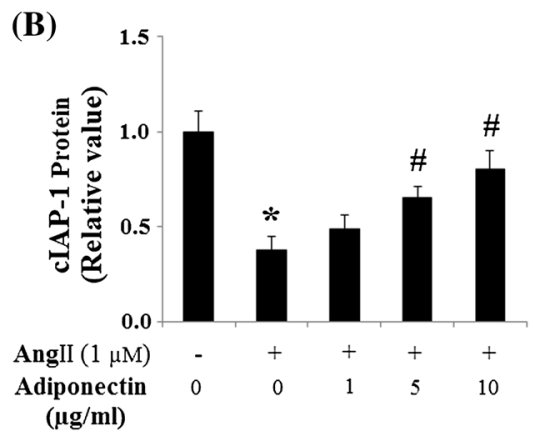

(ANOVA: ${ }^{*} p<0.01$ vs. non-treatment group; $\# p<0.01$ vs. Ang II-treated, but not with the adiponectin-treated group, $n=3$ ) 
(A)

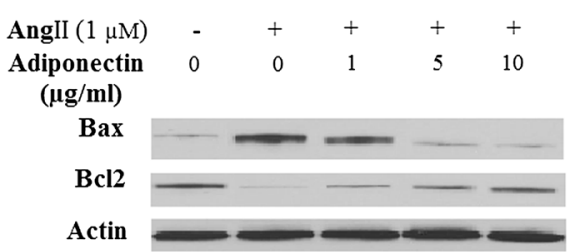

Fig. 11 Effects of adiponectin on the expression of Bax and Bcl-2 in HUVECs. a Representative images of immunoblots for Bax and Bcl-2. The expression of $\beta$-actin was used as a loading control; $\mathbf{b}$ Quantitative
(B)

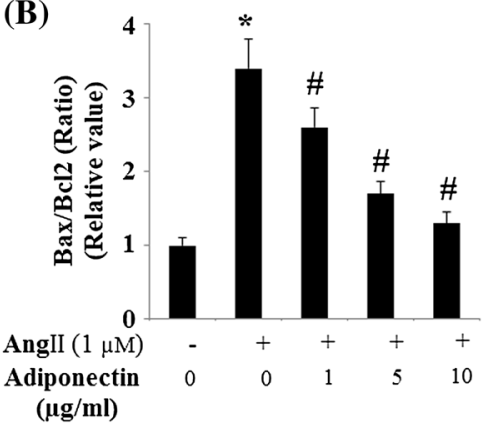

analysis of the ratio of Bax and Bcl-2 (ANOVA * $p<0.01$ vs. the nontreatment group; $\# p<0.01$ vs. the Ang II-treated group, but not with the adiponectin-treated group, $n=3$ ) dysfunction is a key event in the pathology of several cardiovascular and noncardiovascular diseases. Apoptosis of vascular endothelial cells is known to be linked to endothelial dysfunction. Ang II is the main effector of the renin-angiotensin-aldosterone system and has been reported to be involved in various cardiovascular diseases, such as atherosclerosis, hypertension, and heart failure (Nakashima et al. 2006). Mechanically, it has been well documented as being implicated in various pro-inflammatory actions in the vascular wall, including adhesion molecule, chemokine, cytokine expression, and ROS generation (Brasier et al. 2002). Prevention of Ang II toxicity has become an important potential target for treatment in the field of vascular disease. Increased oxidative stress is an important event of Ang II toxicity. Ang II exerts many of its detrimental effects by activating the angiotensin II type 1 receptor, which leads to ROS production and consequent reduction in NO bioavailability.

Adiponectin is the most abundant adipokine in blood. It is reported that the range of blood concentration of adiponectin is between 5 and $30 \mathrm{mg} / \mathrm{ml}$, which varies depending on the assays and subjects used (Scherer 2006). In our study, treatment with adiponectin attenuated oxidative stress induced by Ang II, a result that is consistent with previous studies highlighting adiponectin as a crucial factor in both wholebody energy homeostasis and inhibition of inflammation and oxidative stress. In addition, adiponectin treatment has been shown to produce anti-apoptotic effects, a finding that is also consistent with the present study. Adiponectin causes antiapoptotic effects through various signaling mechanisms. In this study, we found a new underlying mechanism through which adiponectin exerts its anti-apoptotic effects by downregulating the expression of LOX-1. LOX-1 is a lectin-like scavenger receptor for oxidized low-density lipoprotein (oxLDL), expressed primarily in endothelial cells. An array of reports has shown that LOX-1 plays a pivotal role in endothelial apoptosis (Lu et al. 2011; Chen et al. 2002). It is well known that expression of LOX-1 is up-regulated under conditions of oxidative stress and apoptotic cell death as an adaptive mechanism. Up-regulation of LOX-1 induced by Ang II treatment has been previously shown to be the essential regulator of Ang II-mediated pro-inflammatory signaling (Wang et al. 2013). A previous study demonstrated that LOX-1 could mediate ox-LDL-induced endothelial apoptosis via the down-regulation of multiple anti-apoptotic proteins including Bcl-2 and cIAP-1 (Chen et al. 2004; Li and Mehta 2009). Consistent with the current study, adiponectin has been shown to be able to suppress the up-regulation of LOX-1 as well as downregulate Bcl-2 and cIAP-1, which offers insight into a new mechanism in which adiponectin exerts it antiapoptotic effects against Ang II.

Notably, we also proved that APPL1-dependent AMPK activation was involved in the protective effects of adiponectin against Ang II cytotoxicity. APPL1 has been reported to play an important role as an endosomal adaptor protein in various cellular signaling pathways. Essential to activation of AMPK is its phosphorylation at Thr-172 by an upstream kinase. It has been reported that adiponectin is able to activate AMPK by increasing its phosphorylation at Thr-172 (Zhou et al. 2009). On the other hand, the requirement of AMPK for energy-dependent and independent mechanisms of apoptosis induced by Ang II has been previously demonstrated (Day et al. 2011). We also found that Ang II and adiponectin treatment increased the phosphorylated levels of AMPK at Thr-172 (data not shown), which is consistent with previous studies. Importantly, AMPK is an important regulator of cellular energy gauge. AMPK can be activated by an increase in the AMP to ATP ratio. When we inhibited the expression of APPL1 using APPL1 siRNA, the protective effects of adiponectin on the cytotoxicity of Ang II were prevented. Consistently, direct inhibition of AMPK by AMPK siRNA also led to abolishment of the protective effects of adiponectin on the cytotoxicity of Ang II. Our data strongly suggest that active AMPK might play a critical role in the protective effects of adiponectin. 


\section{References}

Akingbemi BT (2013) Adiponectin receptors in energy homeostasis and obesity pathogenesis. Prog Mol Biol Transl Sci 114:317-342

Brasier AR, Recinos A 3rd, Eledrisi MS (2002) Vascular inflammation and the renin-angiotensin system. Arterioscler Thromb Vasc Biol 22:1257-1266

Chen M, Masaki T, Sawamura T (2002) LOX-1, the receptor for oxidized low-density lipoprotein identified from endothelial cells: implications in endothelial dysfunction and atherosclerosis. Pharmacol Ther 95(1):89-100

Chen J, Mehta JL, Haider N et al (2004) Role of caspases in Ox-LDLinduced apoptotic cascade in human coronary artery endothelial cells. Circ Res 94:370-376

Davignon J, Ganz P (2004) Role of endothelial dysfunction in atherosclerosis. Circulation 109(suppl 1):III27-III32

Day RM, Lee YH, Han L et al (2011) Angiotensin II activates AMPK for execution of apoptosis through energy-dependent and -independent mechanisms. Am J Physiol Lung Cell Mol Physiol 301(5):L772-L781

Dimmeler S, Rippmann V, Weiland U et al (1997) Angiotensin II induces apoptosis of human endothelial cells. Protective effect of nitric oxide. Circ Res 81:970-976

He Y, Zhou H, Tang H, Luo Y (2006) Deficiency of disulfide bonds facilitating fibrillogenesis of endostatin. J Biol Chem 281(2):1048-1057

Kadowaki T, Yamauchi T, Kubota N, Hara K, Ueki K et al (2006) Adiponectin and adiponectin receptors in insulin resistance, diabetes, and the metabolic syndrome. J Clin Invest 116:1784-1792

Kim YC, Day RM (2012) Ang II regulates activation of Bim via Rb/E2F1 during apoptosis: involvement of interaction between AMPK $\beta \quad 1 / 2$ and Cdk4. Am J Physiol Lung Cell Mol Physiol 303:L228-L238

Lam KS, Xu A (2005) Adiponectin: protection of the endothelium. Curr Diabet Rep 5(4):254-259

Lee R, Channon KM, Antoniades C (2012) Therapeutic strategies targeting endothelial function in humans: clinical implications. Curr Vasc Pharmacol 10:77-93

Li D, Mehta JL (2009) Intracellular signaling of LOX-1 in endothelial cell apoptosis. Circ Res 104:566-568

Lu J, Mitra S, Wang X, Khaidakov M, Mehta JL (2011) Oxidative stress and lectin-like ox-LDL-receptor LOX-1 in atherogenesis and tumorigenesis. Antioxid Redox Signal 15(8):2301-2333

Mao X, Kikani CK, Riojas RA et al (2006) APPL1 binds to adiponectin receptors and mediates adiponectin signaling and function. Nat Cell Biol 8(5):516-523

Nakashima H, Suzuki H, Ohtsu H, Chao JY, Utsunomiya H, Frank GD, Eguchi S (2006) Angiotensin II regulates vascular and endothelial dysfunction: recent topics of angiotensin ii type-1 receptor signaling in the vasculature. Curr Vasc Pharmacol 4:67-78
Sata M, Fukuda D (2010) Crucial role of renin-angiotensin system in the pathogenesis of atherosclerosis. J Med Invest 57(1-2):12-25

Scherer PE (2006) Adipose tissue: from lipid storage compartment to endocrine organ. Diabetes 55:1537-1545

Sheng B, Gong K, Niu Y et al (2009) Inhibition of gamma-secretase activity reduces Abeta production, reduces oxidative stress, increases mitochondrial activity and leads to reduced vulnerability to apoptosis: implications for the treatment of Alzheimer's disease. Free Radic Biol Med 46(10):1362-1375

Sheng B, Wang X, Su B et al (2012) Impaired mitochondrial biogenesis contributes to mitochondrial dysfunction in Alzheimer's disease. J Neurochem 120(3):419-429

Shi H, Sheng B, Zhang F et al (2013) Kruppel-like factor 2 protects against ischemic stroke by regulating endothelial blood brain barrier function. Am J Physiol Heart Circ Physiol 304(6): H796-H805

Tan NY, Li JM, Stocker R et al (2009) Angiotensin II-inducible smooth muscle cell apoptosis involves the angiotensin II type 2 receptor, GATA-6 activation, and FasL-Fas engagement. Circ Res 105:422430

Wang Y, Lam KS, Yau MH, Xu A (2008) Post-translational modifications of adiponectin: mechanisms and functional implications. Biochem $\mathrm{J}$ 409(3):623-633

Wang L, Hu X, Zhang W, Tian F (2013) Angiotensin (1-7) ameliorates angiotensin II-induced inflammation by inhibiting LOX-1 expression. Inflamm Res 62(2):219-228

Xue H, Zhou L, Yuan P et al (2012) Counteraction between angiotensin II and angiotensin-(1-7) via activating angiotensin type I and Mas receptor on rat renal mesangial cells. Regul Pept 177:12-20

Yamauchi T, Kadowaki T (2008) Physiological and pathophysiological roles of adiponectin and adiponectin receptors in the integrated regulation of metabolic and cardiovascular diseases. Int $\mathrm{J}$ Obes (Lond) 32(Suppl 7):S13-S18

Yang D, Zhang M, Huang X et al (2010) Protection of retinal vasculature by losartan against apoptosis and vasculopathy in rats with spontaneous hypertension. J Hypertens 28:510-519

Zhao HY, Zhao M, Yi TN et al (2011) Globular adiponectin protects human umbilical vein endothelial cells against apoptosis through adiponectin receptor $1 /$ adenosine monophosphate-activated protein kinase pathway. Chin Med J 124:2540-2547

Zhou L, Deepa SS, Etzler JC et al (2009) Adiponectin activates AMPactivated protein kinase in muscle cells via APPL1/LKB1dependent and phospholipase $\mathrm{C} / \mathrm{Ca} 2+/ \mathrm{Ca} 2+/$ calmodulin-dependent protein kinase kinase-dependent pathways. J Biol Chem 284(33): 22426-22435

Zhou G, Hamik A, Nayak L et al (2012) Endothelial Kruppel-like factor 4 protects against atherothrombosis in mice. J Clin Invest 122(12): 4727-4731 